\title{
Perceiving object motion using vision and touch
}

\author{
THOMAS W. JAMES and RANDOLPH BLAKE \\ Vanderbilt University, Nashville, Tennessee
}

\begin{abstract}
In a previous experiment, we showed that bistable visual object motion was partially disambiguated by tactile input. Here, we investigated this effect further by employing a more potent visuotactile stimulus. Monocular viewing of a tangible wire-frame sphere (TS) rotating about its vertical axis produced bistable alternations of direction. Touching the TS biased simultaneous and subsequent visual perception of motion. Both of these biases were in the direction of the tactile stimulation and, therefore, constituted facilitation or priming, as opposed to interference or adaptation. Although touching the TS biased visual perception, tactile stimulation was not able to override the ambiguous visual percept. This led to periods of sensory conflict, during which visual and tactile motion percepts were incongruent. Visual and tactile inputs can sometimes be fused to form a coherent percept of object motion but, when they are in extreme conflict, can also remain independent.
\end{abstract}

There is growing evidence that processing the structural properties of graspable objects is accomplished through a tight coupling of the visual and the somatosensory systems (Easton, Srinivas, \& Greene, 1997; Kilgour, de Gelder, \& Lederman, 2004; Reales \& Ballesteros, 1999). This integration involves not only a sharing of information between the two systems, but also a sharing of neural architecture (Amedi, Jacobson, Hendler, Malach, \& Zohary, 2002; Deibert, Kraut, Kremen, \& Hart, 1999; Hillis, Ernst, Banks, \& Landy, 2002; James et al., 2002; Sathian, Zangaladze, Hoffman, \& Grafton, 1997; Zangaladze, Epstein, Grafton, \& Sathian, 1999). The mounting evidence suggests that regions of the brain that were purportedly visual in nature may, in fact, be bisensory. In a previous report (Blake, Sobel, \& James, 2004), we extended these findings by demonstrating that visual and tactile information were integrated not just during the recognition of static object structure, but also during the discrimination of object motion. Furthermore, we and others (Blake et al., 2004; Hagen et al., 2002) have shown that the motion-selective middle temporal cortex $(\mathrm{MT}+)$ of the human brain is responsive to tactile motion stimulation. Although speculative, these findings suggest that much of the visual cortex and many perceptual functions may, in fact, be bisensory in nature.

In our previous study investigating bisensory motion discrimination (Blake et al., 2004), we used an ambiguous visual motion display to evaluate the effect of tactile input on observers' visual perception. Our assumption was that stimulation by an ambiguous visual motion display would make the visual system more inclined to accept other sen-

This research was supported by NIH Grant EY07760 (to R.B.) and CIHR Grant MFE47716 (to T.W.J.). We thank Karin Harman James, Lee Gilroy, David Bloom, Ken Sobel, Alex Meredith, and two anonymous reviewers for their valuable assistance and comments on this work. Correspondence concerning this article should be addressed to T. W. James, Vanderbilt University, 111 21st Ave. S., Nashville, TN 37203 (e-mail: tom.james@vanderbilt.edu). sory influences. The visual display in our previous study was a two-dimensional projection of dots onto the surface of a virtual globe (VG) rotating about its vertical axis; in the absence of depth cues, the perceived direction of motion (clockwise $[\mathrm{CW}]$ vs. counterclockwise $[\mathrm{CCW}]$ ) fluctuates over time. The tactile stimulus was a three-dimensional styrofoam tactile globe (TG), which could rotate about its vertical axis at the same speed as the VG. We found that tactile input tended to bias visual perception of the direction of rotation, but it could not specify the direction completely; reversals in the direction of motion still occurred despite unambiguous tactile input specifying a given direction of rotation. In other words, the tactile and the visual percepts sometimes became uncoupled under these conditions. This uncoupling was different from that reported in previous studies in which static objects' properties were investigated, in which degrading the information provided by one sense strengthened the contribution made by another but did not lead to uncoupling (Hillis et al., 2002). Furthermore, in our work with rotating globes, only simultaneous visual and tactile inputs interacted; when tactile input preceded visual input, there was no lasting effect of the tactile input. This again was different from results produced with static object stimuli, in which strong effects of cross-sensory perceptual history were found (Easton et al., 1997; Reales \& Ballesteros, 1999). Therefore, our earlier findings (Blake et al., 2004) suggest that the influence of touch on vision may be weaker for discrimination of object motion than for discrimination of object shape.

It is possible that integration was weak in our previous study because the visual stimulation (produced by an array of dots portrayed on a video monitor) and the tactile stimulation (produced by touching a Styrofoam globe) did not arise from the same source. Although care was taken to locate the VG and the TG in the same spatial location, to make them the same size, and to rotate them at the same speed, it was obvious to all the observers that they were touching a stimulus that was different from the one they viewed. One 
especially clear cue, and one that has shown strong effects in previous research (Botvinick \& Cohen, 1998; Làdavas, Farnè, Zeloni, \& di Pellegrino, 2000), was that the observers could not see their own hands. To investigate whether a stronger interaction between vision and touch could be found, we developed an ambiguous visual motion stimulus that could itself also be touched. The stimulus was a wire-frame sphere rotated about its vertical axis that, when viewed monocularly, produced ambiguous (bistable) perception of the two alternative directions of rotation. Monocular viewing conditions with a fixed head position ruled out contributions from stereopsis and motion parallax. In the absence of these potent depth cues, the observers could not readily distinguish near and far wire surfaces of the sphere. This depth ambiguity, in turn, produced ambiguity in the direction of rotation of the sphere as a whole, because direction of rotation $(\mathrm{CW}$ or $\mathrm{CCW}$ ) is calculated by combining depth and direction of translation. Because the observers were able to view the sphere and also touch it, we called it the tangible sphere (TS). Importantly, the observers were able to see their hands touching the TS. We repeated the experiments from our previous study in order to assess the interaction of vision and touch during concurrent and consecutive presentations with this new stimulus. We fully expected that touch, under these conditions, would completely stabilize the perceived direction of visual motion. We expected that simultaneous touching and viewing of the same stimulus, with visual feedback indicating that the viewed stimulus was also being touched, would prevent uncoupling of the tactile and the visual percepts. To our great surprise, however, tactile and visual percepts did become uncoupled from time to time, despite the knowledge that the same object was being touched and viewed.

\section{METHOD}

\section{Participants}

The participants were graduate students, postdoctoral fellows, and research assistants in the psychology department at Vanderbilt University. The experimental protocol was approved by, and consent was received in accordance with, the Institutional Review Board at the Vanderbilt University Medical Center.

\section{Apparatus}

The apparatus consisted of a wire-frame sphere, viewed through an aperture and rotating about its vertical axis (Figure 1). The sphere was constructed of six 5.6-cm-diameter, equally spaced, vertical circular loops attached at the poles. Therefore, the appearance of the sphere was that of the lines of longitude on a globe. The spacing of the wires was approximately $1.5 \mathrm{~cm}$ at the sphere's widest point (equator) when viewed from the front. The wires of the sphere were painted flat black, and the sphere was viewed against a featureless white background. The sphere was rotated in either of two directions about its vertical axis, with a velocity of one revolution every $3.8 \mathrm{sec}$. This speed was chosen because it had produced reliable results in our previous study (Blake et al., 2004), in which, in turn, this speed had been used after careful pilot testing. These findings, although not reported, showed that ambiguity of direction occurred within a large range of rotation speeds and showed marked individual differences across observers. At the viewing distance of $33 \mathrm{~cm}$ used in our present study, the sphere subtended $9.7^{\circ}$ of visual angle. A cardboard screen with a $3 \times 7 \mathrm{~cm}$ aperture was placed between the observer and the sphere at a distance of $15.5 \mathrm{~cm}$ from the observer. The positioning of the aperture was such that the sphere could be viewed fully with either eye but the point of attachment between the sphere and the driving motor was occluded. Viewing conditions were monocular with a fixed head position, which was accomplished with the use of an eye patch and a head-/chinrest. The eye used for monocular viewing was chosen for each observer by a coin flip. The sphere was illuminated using the ambient fluorescent lighting of the testing room.

\section{Procedure}

In Experiment 1, 5 observers ( 3 naive) viewed the TS during 60 -sec observation periods and pressed one of two buttons to indicate from moment to moment their perception of the visual direction of rotation. In the study of ambiguous visual displays, such as binocular rivalry and the kinetic depth effect (Leopold, Wilke, Maier, \& Logothetis, 2002; Nawrot \& Blake, 1989) used here, this tracking procedure is a standard way of measuring the effect of different manipulations on the observer's subjective experience with a stimulus. Successive observation periods alternated randomly between three conditions: (1) visual only (not touching the TS), (2) touching the front of the TS with the pad of the right thumb, and (3) grasping the TS and, thereby, enclosing it with the entire right hand. The veridical direction of rotation ( $\mathrm{CW}$ or $\mathrm{CCW}$ about the vertical axis) of the TS was also randomly determined on each trial (where veridical corresponds to the actual direction of rotation of the TS). The observers began each trial with the unpatched eye closed and opened that eye when given an auditory cue. There were two $\mathrm{CW}$ and two CCW trials for each of the two tactile conditions and double that number for the visual condition. Condition 1, the visual-only condition, was in-
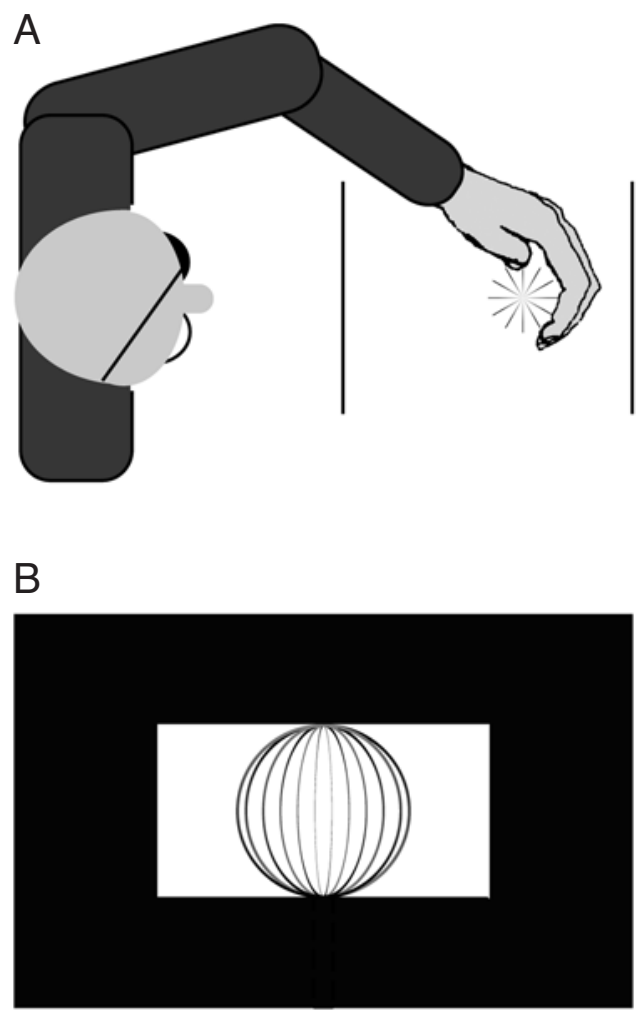

Figure 1. Tangible sphere apparatus. (A) A wire-frame sphere was viewed monocularly with a fixed head position while being touched. (B) The sphere was viewed through an aperture so that its connection to the drive shaft was occluded. 
cluded to assess the visual ambiguity of the display. Approximately equal reporting of $\mathrm{CW}$ and $\mathrm{CCW}$ directions of rotation would indicate complete ambiguity, whereas a bias for reporting the veridical direction of rotation would indicate that monocular cues were influencing perception of the relative depths of the near and far wires.

Experiment 2 was a variant of Experiment 1. Nine observers ( 6 naive) tracked the visual perceived direction of rotation of the TS while touching the back of the TS with one, two, or three fingers from either the left or the right hand. For Experiment 1, we realized that touching the front of the TS with the thumb caused visual occlusion of the TS. Touching the back of the TS with the fingers not only alleviated the occlusion problem, but also allowed parametric variation of the tactile receptor area. There were two $\mathrm{CW}$ and two $\mathrm{CCW}$ trials for each of these six conditions. Our aim here was to vary the magnitude and quality of the tactile input, gauging the effect of this variation on visual perception of the direction of rotation.

Experiment 3 was different from Experiments 1 and 2; instead of tracking the visual direction of rotation, our observers ( 2 naive) reported the direction of rotation in discrete trials. To accomplish this, each trial began with an auditory cue that cued the observer to open his/her eye. The observer then immediately noted the perceived direction of rotation and indicated that direction, using one of two keys on the computer keyboard. It was important for the observers to report the initial perceived direction, because the perceived direction was likely to change over time due to the ambiguity of the visual display (as the results from Experiment 1 revealed). Interspersed between each trial was a 5-sec intertrial interval (ITI). For 20 of the 40 total trials, the observers simply waited with eyes closed during the ITI, and for the other 20 trials, the observers closed their eyes but, at the same time, enclosed the TS with their hands. Because the TS was moving during this 5-sec ITI period, these trials were considered tactile priming trials. The actual direction of rotation of the sphere employed for any given ITI was randomly determined.

Experiment 4 was a variant of Experiment 3. Instead of a 5-sec ITI, there was a 90 -sec ITI. During this time, 5 observers ( 2 naive) either waited with eyes closed or enclosed the moving TS with their hands. After this 90 -sec period, the next trial was initiated by an auditory cue, which signaled the observer to open his/her eyes. At that point, the observers began tracking (as in Experiments 1 and 2) the visually perceived direction of rotation of the TS for $15 \mathrm{sec}$. (It would be too time consuming to collect data on multiple discrete trials, given the 90 -sec ITI, which is why we opted for the tracking task in this experiment.) The veridical direction of rotation of the TS was randomly determined on each trial. There were four $\mathrm{CW}$ and four $\mathrm{CCW}$ trials in each condition. Before trials in which observers touched the TS for $90 \mathrm{sec}$, we inserted a 60 -sec delay, during which the observers neither looked at nor touched the TS. This delay was inserted to ensure that any effect of enclosing the TS on a previous trial would have dissipated before the subsequent trial was commenced.

The preceding four experiments were designed to test the effect of tactile input on visual perception of rotation. Experiment 5 was designed to test the opposite - that is, the effect of visual input on tactile perception of rotation. The key difference with Experiment 5 was that the observers reported the tactilely perceived direction of rotation instead of the visually perceived direction. Three observers ( 2 naive) viewed but did not touch the TS, and they tracked its direction of rotation until the occurrence of an auditory cue. Upon hearing the cue, the observers then touched the TS briefly with one finger while their eyes remained open; they verbally reported their tactile perception of the direction of rotation. Because the observers tracked their visual perception of the TS before touching it, the visual perceptual state of the observer during the touching event was known on each discrete trial. Because the visually perceived direction of rotation was bistable, the TS could appear to rotate in a direction congruent or incongruent with the veridical direction of rotation. There were four $\mathrm{CW}$ and four $\mathrm{CCW}$ trials for each of the congruent and incongruent conditions.

\section{RESULTS}

Experiment 1 had two aims: to assess whether the TS apparatus actually produced ambiguous visual perception of rotation and, if so, to assess whether tactile input would influence the visual perception of rotation. Concerning the first aim, inspection of the tracking data for the visual-only condition revealed that all the observers experienced both directions of rotation during the 60 -sec tracking period, confirming that the TS was indeed ambiguous. There was some tendency for the durations of perception of the veridical direction of rotation to be longer than the durations of the nonveridical direction. A one-sample $t$ test, however, revealed that this trend failed to reach statistical significance $[t(4)=1.65, p<.1]$. There may well have existed some subtle monocular depth cue (e.g., shading) that indicated the veridical direction of rotation of the TS, thus producing this nonsignificant trend. Nevertheless, these visual-only results confirm that the TS was robustly bistable and, therefore, appropriate as a stimulus for the purposes investigated in the remaining experiments.

Turning to the second aim of Experiment 1, we assessed the effect of tactile input on visual perception of rotation, using a one-way analysis of variance (ANOVA) on the data shown in Figure 2A. This analysis showed a significant effect of tactile input $\left[F(2,8)=4.46, M S_{\mathrm{e}}=0.00559, p<\right.$ $.05]$. Post hoc tests computed using Tukey's HSD showed that when the observers enclosed the TS with their hands, they reported the veridical direction of rotation for significantly longer durations than when there was no tactile input $[q(8,3)=4.16, p<.05]$. No other post hoc tests were significant. Figure 3 compares the results of Experiment 1 , in which the TS was used, with the results of our previous study (Blake et al., 2004), in which the VG was used. This comparison is interesting because, with the TS, the observers saw their hands touching the same stimulus as the one they were viewing. Despite this difference between the TS and the VG displays, it is clear that the pattern of results was not different and that tactile input with the TS did not produce a stronger bias than did tactile input with the VG. The equivalence of these two conditions is rather surprising, given that the observers in the present experiment, unlike those in the earlier experiment, knew that they were viewing the same stimulus as the one they were touching.

Another result from Experiment 1 was that the bias toward veridical perception of the TS appears larger when the TS was enclosed with the whole hand, as opposed to being touched with only the pad of the thumb (Figure 2A). This effect, however, was not significant when tested with Tukey's HSD $[q(8,3)=3.66, p>.05]$. Therefore, to explore this finding further, we conducted Experiment 2, in which the observers touched the TS with one, two, or three fingers. Our aim was to learn whether the improved tactile input afforded by multiple fingers would produce a stronger influence over visual perception of motion direction. Like the results of Experiment 1, the results of Experiment 2 showed that touching the TS biased visual per- 

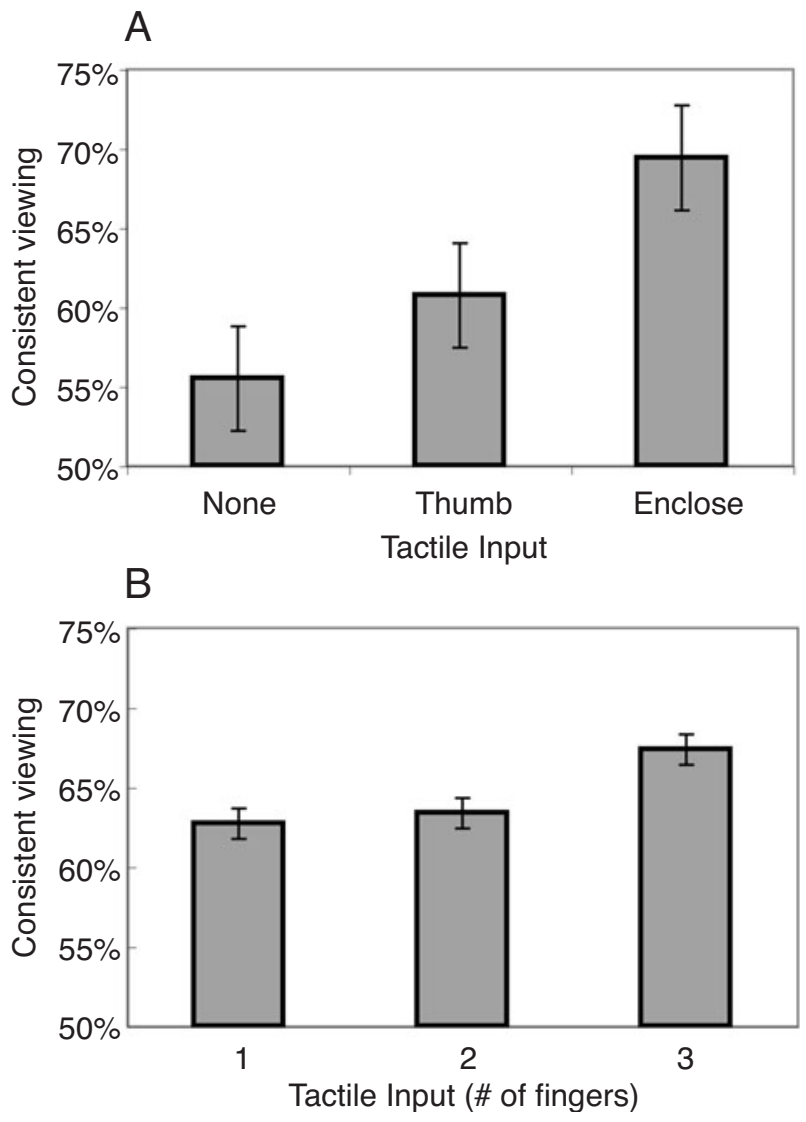

Figure 2. Tracking durations as a function of tactile input condition. The vertical axis represents the percentage of time the tangible sphere was visually perceived as rotating in the veridical direction. Error bars are the square root of the $M S_{\mathrm{e}}$. (A) Data from Experiment 1. (B) Data from Experiment 2.

ception to be more veridical (Figure 2B). A one-way ANOVA also showed a significant effect of the number of digits used $\left[F(2,16)=7.19, M S_{\mathrm{e}}=0.000823, p<.05\right]$. Post hoc tests using Tukey's HSD showed that touching the TS with three fingers produced a larger bias on visual perception than did touching the TS with one finger $[q(16,3)=4.85, p<.01]$ or with two fingers $[t(16,3)=$ $4.18, p<.05]$.

In Experiments 1 and 2, we investigated the effect of simultaneous tactile input on visual perception of an ambiguous motion display. Experiments 3 and 4 were designed to investigate the effect of tactile preexposure to the TS on subsequent visual perception of the TS. Five seconds of tactile preexposure to the TS immediately preceding visual presentation produced a significant bias toward veridical visual perception of motion direction, as compared with no tactile input $[t(3)=3.54, p<.05]$. Figure 4 compares the results of Experiment 3 with the results from our previous study. The number of discrete trials in which visual perception was consistent with tactile perception is plotted as a function of preexposure time and study. The significant priming effect found in Experiment 4 was not found in our previous study, in which touching the TG had no effect on subsequent viewing of the VG.

The results of Experiment 4 also differed from those in our previous study. In Experiment 4, the preexposure duration was increased to $90 \mathrm{sec}$. With such a long preexposure period, our expectation was that any influence would, in fact, result in adaptation, instead of priming, producing a shift in visual perception away from the veridical direction. Ninety seconds of tactile preexposure to the TS did produce a significant bias $[t(4)=7.35, p<.001]$, but contrary to our expectation, the bias was toward veridical visual perception. In other words, both 5 and $90 \mathrm{sec}$ of tactile preexposure produced facilitation of visual perception, in that visual perception of rotation was biased toward the veridical direction.

Experiments 1-4 were concerned with the influence of tactile input on visual perception. Debriefing after these experiments revealed that the participants did not experience periods of time when tactile perception was bistable. This implies, then, that there were periods of time during which the visual and the tactile perceptual experiences were at odds even though the observers knew they were touching the same object as the one they were seeing. Tactile perception, in other words, seemed to influence bistable visual perception, but bistable visual perception could not influence tactile perception.

Experiment 5 tested this notion explicitly by testing whether nonveridical visual perception of the TS could affect tactile perception of the TS. Instead of tracking the visually perceived direction of rotation of the TS, the observers tracked the perceived direction of rotation experienced by touching the TS. Despite reporting afterward that their visual perception of rotation was still ambiguous and, therefore, alternated between veridical and nonveridical directions, the observers were $100 \%$ accurate at reporting the tactile perceived (or veridical) direction of the TS. Therefore, under these conditions, tactile percep-

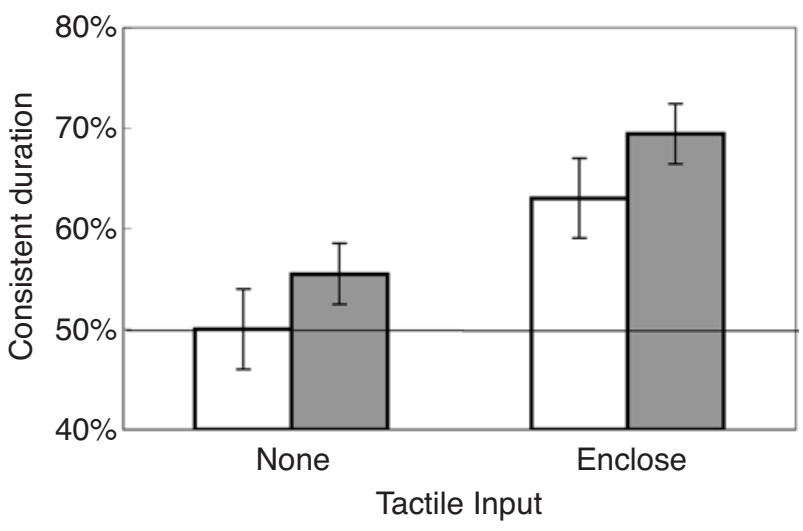

Figure 3. A comparison of Experiment 1 with previous data. The white bars represent previous data, and the gray bars represent the data from Experiment 1. The vertical axis represents the percentage of time the tangible sphere was visually perceived as rotating in the veridical direction. Error bars are standard errors of the means. The horizontal line at $50 \%$ represents chance performance (i.e., no bias). 


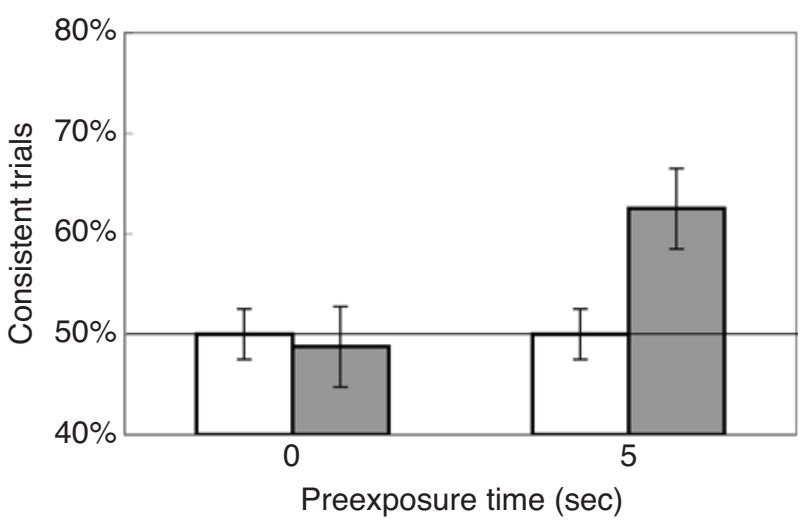

Figure 4. The influence of tactile priming on perceived direction. The white bars represent previous data, and the gray bars represent the data from Experiment 3. The vertical axis represents the percentage of trials on which the reported initial direction of rotation was consistent with the veridical direction. For the previous data, veridical was taken to be the direction of rotation of the tactile globe for the 5-sec condition and was taken as clockwise for the 0 -sec condition. The horizontal line at $50 \%$ represents chance performance (i.e., no bias).

tion of motion not only was uncoupled from visual perception, but also was resistant to influence by visual perception. In this way, tactile perception always indicated the true rotation direction of the TS. This was a reasonable expectation, because visual reversals occurred only as a result of the monocular viewing conditions, which stripped the scene of binocular depth cues. We made no provision to limit the depth information available to touch; therefore, the tactile percept should have accurately described the direction of motion, which it did.

\section{DISCUSSION}

The present study was an extension of previous work (Blake et al., 2004) in which multisensory perception of object motion was evaluated. A comparison of the results from our previous study and those from the present experiments showed both similarities and differences. In the present study, the observers viewed and touched the same stimulus (the TS) and, importantly, were able to view their own hands touching the stimulus. In the previous experiment, the observers viewed one stimulus (the VG), but touched a different stimulus (the TG) and could not view their hands touching the TG. Our previous study was conducted with separate visual and tactile stimuli in order to maximize our ability to place the two stimulus modalities in conflict; it also allowed greater control of the visual display features, because it was a video display controlled by a computer program. In the present experiment, we sacrificed this level of control to test whether viewing and touching the same stimulus would produce stronger effects than those in our previous study.

The findings from Experiments 1 and 2 of the present study, in which simultaneous visual and tactile input was examined, were consistent with the findings from the pre- vious study but did not show stronger effects. In that study, enclosing the TG while viewing the VG caused observers to report seeing the VG rotate in the direction of the TG for longer durations. Likewise, in Experiments 1 and 2, enclosing the TS or touching it with one, two, or three digits caused the observers to report the veridical direction for longer durations. Contrary to our expectations, using the TS did not increase the effect of the tactile input over that shown in the previous study. This suggests that for simultaneous viewing and touching of a stimulus, the exact stimulus properties are not important. In our previous study, we went to great lengths to make the VG and the TG as similar as possible. To accomplish this, we used a specially designed mirror stereoscope to place the VG at the same apparent location as the TG, and we made sure that the size and texture of the TG matched the size and dot texture of the VG. Perhaps these similarities produced enough congruence for tactile input from the TG to affect visual perception of the VG to the same degree as that shown here with the TS.

In Experiments 3 and 4, we examined sequential touching and viewing of the TS. The results of these experiments were different from those in our previous study. In that study, the influence of touch on vision was present only during simultaneous stimulation. Touching of the TG had no influence on subsequent viewing of the VG, despite the fact that our previous study showed that visual adaptation does occur when a VG is preceded by another VG (Figure 5). By adaptation, we mean that there was a bias to see the VG rotate in the direction opposite to that of the preceding VG. In Experiments 3 and 4, we examined the effect of tactile preexposure with the TS on subsequent visual perception of the TS. Even though these two ex-

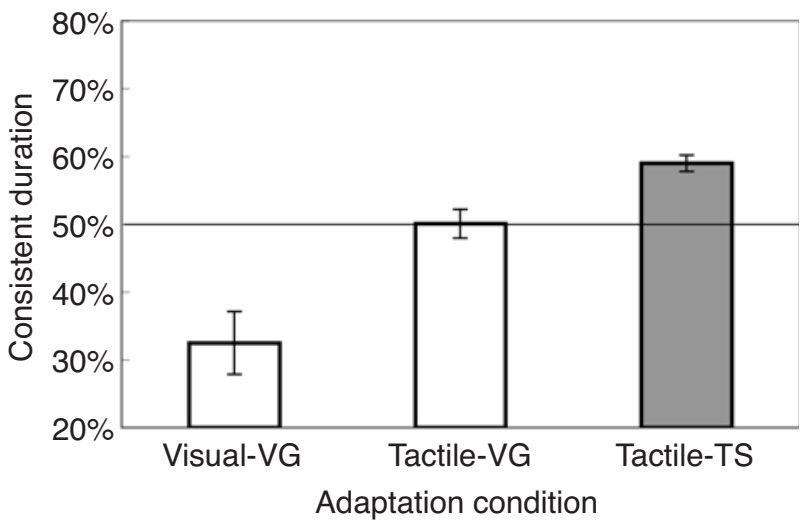

Figure 5. The influence of tactile adaptation on perceived direction. The white bars represent previous data, and the gray bar represents the data from Experiment 4. The vertical axis represents the percentage of time that the tangible sphere (TS) or the virtual globe (VG) was visually perceived as rotating in the veridical direction. For the previous data, veridical was taken to be the direction of rotation of the tactile globe for the tactile condition or the stereoscopic VG for the visual condition. The horizontal line at $50 \%$ represents chance performance (i.e., no bias). Bars below the line indicate adaptation or habituation to the preexposed direction, whereas bars above the line indicate entrainment or priming to the preexposed direction. 
periments used substantially different preexposure times ( 5 vs. $90 \mathrm{sec}$ ), they showed similar effects. But this effect was not adaptation; instead, preexposure caused subsequent viewing of the TS to be biased in the same direction as the one used during preexposure. This facilitatory type of effect is usually termed priming; that is, subsequent visual perception of the TS was primed by preexposure and, therefore, was seen more often to rotate in the same direction as the preexposed TS. The fact that visual perception of the TS was influenced by tactile preexposure in the present experiment suggests that the TS may, in fact, be a more potent stimulus than the combination of a TG and a VG used in our previous experiment, in which only simultaneous exposure produced integration of the multisensory inputs. It is interesting, though, that lengthy tactile preexposure produced a priming effect similar to that with a short preexposure. As was stated above, previous research has shown that lengthy visual preexposure causes adaptation, not priming. Perhaps the TS was sufficiently potent to prime the visual system but not sufficiently potent to produce significant adaptation. Alternatively, the TS may be a more potent stimulus than the combination of VG and TG and, therefore, did not require simultaneous exposure to show integration of visual and tactile inputs. But tactile input may affect different aspects of the visual system than does visual input, and tactile input was perhaps unable to cause adaptation of the visual motion perception system.

Together, Experiments 1 and 2 demonstrate that the amount or quality of tactile input was important during integration of visual and tactile motion perception. We did not manipulate grasp characteristics in our previous experiment - when touching the TG, the observers always enclosed it with both hands. In the present experiment, enclosing the TS produced a stronger bias than did touching with the thumb only and touching the TS with three fingers produced a stronger bias than did touching with one or two fingers. It is difficult to attribute these effects to high-level cognitive factors. For instance, in Experiment 5, we showed that the veridical direction of the TS was easily determined with tactile input from only one digit. A cognitive or nonsensory bias on the visual perceived direction should be based solely on knowing the direction and should be invariant with the quality or amount of tactile information (given that that amount is sufficient to establish the direction). Therefore, if the tactile bias of visual motion perception were due only to knowledge of that direction, the bias would not increase when three fingers versus one were used. We suggest, then, that the tactile bias of visual motion perception may be due to a direct somatosensory input to visual motion centers, instead of a more indirect top-down influence.

The results reported here, combined with those of our previous study (Blake et al., 2004), show that visual information and tactile information are integrated to perform judgments of object motion direction. One expectation was that having observers touch the same object as the one they were viewing would increase the potency of the interaction between vision and touch. In Experiments 1 and 2, in which the observers were able to see their hands touching the sphere, the bias for visual perception was not changed. Debriefing did reveal, however, that the subjective experience with the TS was quite different from that with the VG. Inconsistency in the visual and the tactile (veridical) directions of rotation of the TS created a feeling that the observers characterized as "weird" and "freaky." This kind of perceptual dissonance was not reported in our previous study when the directions of the VG and the TG were inconsistent. Therefore, there is subjective evidence that the TS was a more potent stimulus than the VG/TG but, despite the perceptual dissonance that inconsistent viewing produced, touching the TS was unable to specify unambiguously the visually perceived direction of rotation. This striking effect suggests that the mechanism underlying bistable perception of the direction of rotation of the TS must exert an enormous influence on the visual system, if it cannot be reversed by reliable veridical tactile input.

It is worthwhile stating that the resistance of visual perception to tactile stimulation in these experiments was not due to a dominance of vision over touch, which would lead to alternations in the tactile percept over time that would follow the visual alternations. On the contrary, as was demonstrated in Experiment 5, tactile perception of motion direction was immune to the influence of incongruent visual perception. In other words, as the visual percept alternated over time, the tactile percept remained veridical, and the participants were able to maintain separate visual and tactile percepts. Thus, instead of visual dominance over touch, the inability of tactile stimulation to halt the perceptual alternations of the ambiguous visual display seems more attributable to a decoupling of visual and tactile motion perception during periods of perceptual conflict. Therefore, although visual and tactile inputs are sometimes integrated to form a coherent percept of object motion, when they are in conflict, they can also remain independent. Despite the common misconception that situations of sensory conflict occur in the laboratory only, there are several examples of natural cross-sensory incongruence (de Gelder \& Bertelson, 2003). Therefore, a decoupling of visual and tactile motion perception during incongruent stimulation may be adaptive for both systems. But, in situations in which visual and tactile information are congruent, the nervous system appears to integrate information to arrive at a more accurate prediction of the veridical state of the environment. These findings, together with other work on visual/tactile interactions, underscore the notion that perception of objects, whether stationary or moving, is multisensory in nature, even for creatures as strongly visual as humans.

\section{REFERENCES}

Amedi, A., Jacobson, G., Hendler, T., Malach, R., \& Zohary, E. (2002). Convergence of visual and tactile shape processing in the human lateral occipital complex. Cerebral Cortex, 12, 1202-1212. Blake, R., Sobel, K. V., \& James, T. W. (2004). Neural synergy between kinetic vision and touch. Psychological Science, 15, 397-402. Botvinick, M., \& Cohen, J. (1998). Rubber hands "feel" touch that eyes see. Nature, 391, 756. 
DE Gelder, B., \& Bertelson, P. (2003). Multisensory integration, perception and ecological validity. Trends in Cognitive Sciences, 7, 460-467.

Deibert, E., Kraut, M., Kremen, S., \& Hart, J. J. (1999). Neural pathways in tactile object recognition. Neurology, 52, 1413-1417.

Easton, R. D., Srinivas, K., \& Greene, A. J. (1997). Do vision and haptics share common representations? Implicit and explicit memory within and between modalities. Journal of Experimental Psychology: Learning, Memory, \& Cognition, 23, 153-163.

Hagen, M. C., Franzen, O., McGlone, F., Essick, G., Dancer, C., \& PARdo, J. V. (2002). Tactile motion activates the human middle temporal/V5 (MT/V5) complex. European Journal of Neuroscience, 16, 957-964.

HiLlis, J. M., ERnst, M. O., Banks, M. S., \& LANDY, M. S. (2002). Combining sensory information: Mandatory fusion within, but not between, senses. Science, 298, 1627-1630.

James, T. W., Humphrey, G. K., Gati, J. S., Servos, P., Menon, R. S., \& Goodale, M. A. (2002). Haptic study of three-dimensional objects activates extrastriate visual areas. Neuropsychologia, 40, 1706-1714.

Kilgour, A. R., De Gelder, B., \& Lederman, S. J. (2004). Haptic face recognition and prosopagnosia. Neuropsychologia, 42, 707-712.
LÀdavas, E., Farnè, A., Zeloni, G., \& di Pellegrino, G. (2000). Seeing or not seeing where your hands are. Experimental Brain Research, 131, 458-467.

LEOPOLD, D. A., Wilke, M., Maier, A., \& Logothetis, N. K. (2002). Stable perception of visually ambiguous patterns. Nature Neuroscience, 5, 605-609.

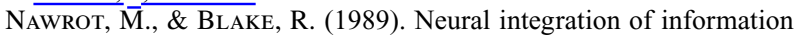
specifying structure from stereopsis and motion. Science, 244, 716-718.

Reales, J. M., \& Ballesteros, S. (1999). Implicit and explicit memory for visual and haptic objects: Cross-modal priming depends on structural descriptions. Journal of Experimental Psychology: Learning, Memory, \& Cognition, 25, 644-663.

Sathian, K., Zangaladze, A., Hoffman, J. M., \& Grafton, S. T. (1997). Feeling with the mind's eye. NeuroReport, 8, 3877-3881.

Zangaladze, A., Epstein, C. M., Grafton, S. T., \& Sathian, K. (1999). Involvement of visual cortex in tactile discrimination of orientation. Nature, 401, 587-590.

(Manuscript received October 4, 2003; revision accepted for publication April 22, 2004.) 